hep-th/0701235 v3

\title{
Hawking radiation from the dilatonic black holes via anomalies
}

\author{
Qing-Quan Jiang ${ }^{1) *}$, Shuang-Qing $\mathrm{Wu}^{2) \dagger}$, and $\mathrm{Xu} \mathrm{Cai}^{1) \ddagger}$ \\ 1) Institute of Particle Physics, Central China Normal University, \\ Wuhan, Hubei 430079, People's Republic of China \\ ${ }^{2)}$ College of Physical Science and Technology, Central China Normal University, \\ Wuhan, Hubei 430079, People's Republic of China
}

\begin{abstract}
Recently, Hawking radiation from a Schwarzschild-type black hole via gravitational anomaly at the horizon has been derived by Robinson and Wilczek. Their result shows that, in order to demand general coordinate covariance at the quantum level to hold in the effective theory, the flux of the energy momentum tensor required to cancel gravitational anomaly at the horizon of the black hole, is exactly equal to that of $(1+1)$-dimensional blackbody radiation at the Hawking temperature. In this paper, we attempt to apply the analysis to derive Hawking radiation from the event horizons of static, spherically symmetric dilatonic black holes with arbitrarily coupling constant $\alpha$, and that from the rotating Kaluza-Klein $(\alpha=\sqrt{3})$ as well as the Kerr-Sen $(\alpha=1)$ black holes via an anomalous point of view. Our results support Robinson-Wilczek's opinion. In addition, the properties of the obtained physical quantities near the extreme limit are qualitatively discussed.
\end{abstract}

PACS numbers: 04.60.-m, 04.62.+v, 04.70.Dy, 11.30.-j

\section{INTRODUCTION}

Since Stephen Hawking proved that a black hole can emit any kinds of particles from its event horizon with a temperature proportional to its surface gravity, many derivations of Hawking radiation had been proposed [1, 2, 3, 4] during the past thirty years. Among them, some tied Hawking radiation to the contributions of the anomalies in different symmetries. For example, Christensen and Fulling [5] showed that the anomaly in the conformal symmetry could give strong constraints on the trace of the energy momentum tensor, and Hawking radiation arose from the contribution of the trace anomaly of the energy momentum tensor [6]. Imposing the $(1+1)$ dimensional anomalous trace equation everywhere in the whole spacetime, a freely falling observer would observe an outgoing flux, which is in quantitative agreement with Hawking's original result. This seems to be a neat derivation of Hawking radiation, however, their observation was based on several assumptions: Firstly, the background was in $(1+1)$ dimensions. Secondly, the fields were massless. Finally, there were no back-scattering effect. Therefore, their derivation appeared to be a rather special phenomenon.

Recently, Robinson and Wilczek [7] proposed a new derivation of Hawking radiation from a Schwarzschildtype black hole via the anomaly in general coordinate symmetry at the horizon, namely via gravitational anomaly. In their opinion, Hawking radiation is effectively treated as the required flux of the energy momentum tensor to cancel gravitational anomaly and to restore general coordinate covariance at the quantum

\footnotetext{
*E-mail address: jiangqingqua@126.com

$\dagger$ E-mail address: sqwu@phy.ccnu.edu.cn

‡E-mail address: xcai@mail.ccnu.edu.cn
}

level. In fact, the idea of this effective theory stems from the choice of vacuum states. Normally, a static, spherically symmetric black hole without a positive cosmological constant has a global Killing vector, but it is only timelike in the region outside the horizon of the black hole. Thus, if one associates the positive energy with the occupation of modes of a positive frequency, there would be a divergent energy momentum tensor due to a pile up of horizon-skimming modes at the horizon since this time coordinate becomes mathematically ill-defined at the horizon. Replacing the coordinates $(t, r)$ with the Kruskal extension $(U, V)$, then the final form of the spacetime showed that $U \rightarrow U+U_{0}$ is an isometry along the past horizon $V=0$, where $\xi \equiv \partial_{U}$ is a Killing vector.

As is well-known, there are various different ways to define the vacuum state in curved spaces. Unruh [8] introduced a quantum vacuum state, the so-called Unruh state or $\xi$-vacuum, for the static spacetime by defining positive energy states as those that have positive frequency with respect to $\xi$ on the past horizon, and letting this vacuum propagate outward and forward in time. The vacuum state defined in this way differs from the one obtained by defining positive energy with respect to the global Killing vector $\eta$, which is called the Boulware state, or $\eta$ vacuum [9]. The $\eta$-vacuum has divergences in its energymomentum tensor arising from horizon-skimming modes, despite appearing empty to static observers. This divergence renders the Boulware state an unphysical candidate vacuum. On the other hand, the Unruh state is well behaved at the horizon with respect to the Kruskal coordinate $U$. In addition, Unruh found that the $\xi$-vacuum is exactly a thermal ensemble of the Boulware states at Hawking temperature $T=\kappa /(2 \pi)$. If elevating the choice of a state to the level of a choice of the theory, one expects that the effective theory should be formulated to exclude the offending modes at the horizon, and describe a thermal flux, in exact agreement with the earlier results of Hawking [1]. 
Since the effective field theory is formulated outside the horizon to integrate out the horizon-skimming modes, it becomes chiral there, and suffers from gravitational anomaly because the numbers of the ingoing and outgoing modes are no longer identical there. To demand general coordinate covariance at the quantum level to hold in the effective theory, one must introduce a compensating flux of the energy momentum tensor to cancel gravitational anomaly at the horizon of the black hole. As a result, the energy momentum tensor flux, which is required to cancel gravitational anomaly and to restore general coordinate covariance at the quantum level, is exactly equal to that of $(1+1)$-dimensional blackbody radiation at the Hawking temperature.

A key technique in the Robinson-Wilczek's method is to reduce the higher dimensional theory to the twodimensional case, in which gravitational anomaly appears as a chiral one. If each partial wave of the higherdimensional theory behaves, near the black hole horizon, as a $(1+1)$-dimensional blackbody source at the Hawking temperature, one reproduces the standard calculation of Hawking radiation. Obviously because the derivation of Hawking radiation via the anomalous point of view is only dependent on the information of the horizon of the black hole, it is more universal than that from the trace anomaly. Following this method, a lot of work that generalizes to various black hole cases appeared [10, 11, 12, 13] in recent months.

In the case of a charged black hole [10], one should also consider the conservation of electric charge beyond the energy conservation. In such case, the two-dimensional theory for each partial wave contains both gauge and general coordinate symmetries, where the gauge symmetry arises from the gauge potential with respect to the electric field of the original charged black hole. Near the horizon, when omitting the classically irrelevant ingoing modes, the effective near-horizon quantum field becomes chiral, and contains the anomalies related to these two symmetries, also known as gauge or gravitational anomalies. To demand gauge invariance and general coordinate covariance at the quantum level, two kinds of compensating fluxes must be introduced to cancel the corresponding gauge and gravitational anomalies at the horizon. Thee flux used to cancel the gauge anomaly is called as the charge current flux, and the other one is the energy momentum tensor flux responsible for cancelling the gravitational anomaly.

As far as the case of a rotating black hole is concerned, one should take into account the conservation of angular momentum. After reduction to the $(1+1)$ dimensions, each partial wave now contains a new $U(1)$ gauge symmetry besides the general coordinate symmetry. Although this $U(1)$ gauge symmetry originates from the isometry along $\varphi$-direction, it can be viewed as arising from the $U(1)$ gauge potential if written in terms of the angular velocity at the horizon of the rotating black hole, in which the $U(1)$ gauge charge $m$ is an azimuthal quantum number. This means that the two-dimensional reduction of the rotating black hole can be equivalently treated as that of a charged particle in a two-dimensional charged field. As such, the same procedure as that in the charged case can be applied to obtain the fluxes required to cancel $U(1)$ gauge and gravitational anomalies at the horizon of the rotating black hole 11, 12, 13]. In order to demand gauge invariance and general coordinate covariance at the quantum level, the fluxes derived in both cases via anomalies must be equal to that of $(1+1)$-dimensional blackbody radiation with the Planck distribution, including chemical potentials for an electric charge $e$ or an azimuthal angular momentum $m$ of the scalar fields radiated from the black holes.

Since the dilatonic black holes [14, 15, 16, 17, 18] obtained from the low energy effective field theory have qualitatively different properties from those in ordinary Einstein gravity, it is necessary and helpful to study the thermal properties of these black holes via the anomalous point of view. In this paper, motivated by RobinsonWilczek's recent viewpoint, we study Hawking radiation from the static, spherically symmetric dilatonic black holes with arbitrary coupling constant, and that from the rotating Kaluza-Klein and Kerr-Sen dilatonic black holes [19] via gauge and gravitational anomalies. The result shows that, demanding gauge invariance or general coordinate covariance at the quantum level, one can find each partial wave of the scalar field to be in a state with a net charge current or energy momentum tensor flux, whose values are exactly equal to that of $(1+1)$-dimensional blackbody radiation at the Hawking temperature with an appropriate chemical potential.

The paper is outlined as follows. We begin with our studies in Sec. III by applying the Robinson-Wilczek's method of anomaly cancellation to study Hawking radiation from the static, spherically symmetric dilatonic black holes with arbitrary coupling constant. Sec. III and Sec. IV]are, respectively, devoted to investigating the cases of the rotating Kaluza-Klein and Kerr-Sen dilatonic black holes in four dimensions via gauge and gravitational anomalies. Sec. V]ends up with some discussions. In addition, special properties of these dilatonic black holes in the extreme limit are also qualitatively discussed.

\section{HAWKING RADIATION FROM THE STATIC, SPHERICALLY SYMMETRIC DILATONIC BLACK HOLE}

The action describing the dilaton field coupled to a $U(1)$ gauge field in $(3+1)$ dimensions is

$$
S=\frac{1}{16 \pi} \int d^{4} x \sqrt{-g}\left[R-2(\nabla \phi)^{2}-e^{-2 \alpha \phi} F^{2}\right],
$$

where $\phi$ and $F$ are the dilaton field and the $U(1)$ gauge field, respectively, with a coupling constant $\alpha$. From the action, the metric of the four-dimensional static, spherically symmetric dilatonic black hole (with an arbitrary 
coupling constant $\alpha$ ), the dilaton field, and Maxwell field had been obtained as [14, 15, 16]

$$
\begin{aligned}
d s^{2} & =-\frac{\Delta}{R^{2}} d t^{2}+\frac{R^{2}}{\Delta} d r^{2}+R^{2}\left(d \theta^{2}+\sin ^{2} \theta d \varphi^{2}\right), \\
\phi & =\frac{\alpha}{1+\alpha^{2}} \ln \left(1-\frac{r_{-}}{r}\right), \\
F & =\frac{Q}{r^{2}} d t \wedge d r
\end{aligned}
$$

where

$$
\Delta=\left(r-r_{+}\right)\left(r-r_{-}\right), \quad R=r\left(1-\frac{r_{-}}{r}\right)^{\alpha^{2} /\left(1+\alpha^{2}\right)},
$$

in which the outer and inner horizons are, respectively, given by

$$
r_{ \pm}=\frac{1+\alpha^{2}}{1 \pm \alpha^{2}}\left[M \pm \sqrt{M^{2}-\left(1-\alpha^{2}\right) Q^{2}}\right] .
$$

For $\alpha \neq 0, r=r_{-}$is the curvature singularity. In the extreme limit (namely, $Q_{\max }=\sqrt{1+\alpha^{2}} M$ ), the inner horizon and the outer horizon coincide with each other, a naked singularity then appears at $r=r_{+}$, and the area of black hole vanishes for $\alpha \neq 0$, the solution no longer represents a black hole.

After performing the conformal transformation $\widetilde{g}_{\mu \nu}=$ $e^{-2 \alpha \phi} g_{\mu \nu}$, the line element (2) for the black hole becomes

$$
d \widetilde{s}^{2}=-f(r) d t^{2}+\frac{1}{g(r)} d r^{2}+r^{2}\left(d \theta^{2}+\sin ^{2} \theta d \varphi^{2}\right),
$$

where

$$
f(r)=\frac{\Delta}{R^{2}} e^{-2 \alpha \phi}, \quad g(r)=\frac{\Delta}{R^{2}} e^{2 \alpha \phi} .
$$

Now, we focus on investigating Hawking radiation from the dilatonic black hole via gauge and gravitational anomalies at the horizon. Near the black hole horizon, the effective radial potential for partial wave modes of the scalar field vanishes exponentially fast if one introduces the tortoise coordinate defined by $r_{*}=\int\left(R^{2} / \Delta\right) d r$, and performs the partial wave decomposition in terms of the spherical harmonics. Thus, the physics near the horizon of the originally four-dimensional black hole can be effectively described by an infinite collection of $(1+1)$ dimensional fields. In the effective two-dimensional reduction, the partial wave is in the background of the metric and the gauge potential

$$
g_{t t}=-f(r), \quad g_{r r}=\frac{1}{g(r)}, \quad A_{t}=-\frac{Q}{r} .
$$

In addition, the background includes a dilaton field, but its contribution can be dropped since the effect of dilaton field does not change the property of the $T_{t}^{r}$ component of the energy momentum tensor in the static spacetime and accordingly the compensating flux is independent of the dilaton background [11].
In the following, we firstly study the flux of the electric current and gauge anomaly at the horizon. In the two-dimensional theory, the electric current is given by integrating the four-dimensional current over a twodimensional sphere. We define the effective field theory outside the horizon. In the region $r_{+}+\epsilon \leq r$, there is no anomalies, so the current satisfies the conservation equation $\partial_{r}\left[\sqrt{-g_{2}} J_{(o)}^{r}\right]=0$. Near the horizon $r_{+} \leq r \leq r_{+}+\epsilon$, when excluding the near-horizonskimming modes whose contributions would give a divergent electric current at the horizon, the effective theory becomes chiral there, and contains the anomaly with respect to gauge symmetry, which originates from the electric field of the charged black hole, also named as gauge anomaly. Thus, the current there obeys the anomalous equation $\partial_{r}\left[\sqrt{-g_{2}} J_{(H)}^{r}\right]=e^{2} \partial_{r} A_{t} /(4 \pi)$. What needs to say is that, in the two-dimensions, the anomalous current actually satisfies $\nabla_{\mu} J^{\mu}=-e^{2} \epsilon^{\mu \nu} \partial_{\mu} A_{\nu} /\left(4 \pi \sqrt{-g_{2}}\right)$. However, one only needs to investigate the electric current of the component $\mu=r$, since the temporal component of the electric current is irrelevant for the Hawking radiation flux of the charge.

In the classical theory, gauge symmetry of the action is expressed by the covariant conservation of the charge current under gauge transformations. However, in the quantized version, the modes' propagation along one lightlike direction makes the time coordinate ill-defined at the horizon for an observer outside the horizon. To remove these offending modes, the current exhibits an anomaly in gauge symmetry. Under gauge transformations, if we integrate the outgoing modes to obtain the effective action, then it changes as $-\delta W=\int d t d r \lambda \nabla_{\mu} J^{\mu}$, where the current is written as $J^{\mu}=J_{(o)}^{\mu} \Theta_{+}(r)+J_{(H)}^{\mu} H(r)$, in which $\Theta_{+}(r)=\Theta\left(r-r_{+}-\epsilon\right)$ and $H(r)=1-\Theta_{+}(r)$ are the scalar step and top hat functions, respectively. Thus the variation of the effective action under gauge transformations is given by

$$
\begin{aligned}
-\delta W= & \int d t d r \lambda\left\{\partial_{r}\left[\frac{e^{2}}{4 \pi} A_{t} H(r)\right]+\left[\frac{e^{2}}{4 \pi} A_{t}\right.\right. \\
& \left.\left.+\sqrt{-g_{2}}\left(J_{(o)}^{r}-J_{(H)}^{r}\right)\right] \delta\left(r-r_{+}-\epsilon\right)\right\},
\end{aligned}
$$

where the ingoing modes are integrated out. To require the full quantum theory gauge invariance, the quantum effect of the omitted ingoing modes should be taken into account. Since its contribution to the total current flux is $-e^{2} A_{t} H(r) /(4 \pi)$, the first term in Eq. (7) is cancelled by its quantum effect [11]. To demand gauge invariance at the quantum level to hold in the effective theory, the coefficient of the delta function should be nullified, which means that

$$
c_{o}=c_{H}-\frac{e^{2}}{4 \pi} A_{t}\left(r_{+}\right),
$$

where we have used the currents in both regions: $\sqrt{-g_{2}} J_{(o)}^{r}=c_{o}$, and $\sqrt{-g_{2}} J_{(H)}^{r}=c_{H}+e^{2}\left[A_{t}(r)-\right.$ $\left.A_{t}\left(r_{+}\right)\right] /(4 \pi)$, in which $c_{o}$ and $c_{H}$ are the values of the 
current at the infinity and the horizon, respectively. In order to fix the current flux, we impose a constraint that demands the covariant current defined by $\widetilde{J}_{(H)}^{r}=$ $J_{(H)}^{r}+e^{2} A_{t}\left(r_{+}\right) /\left(4 \pi \sqrt{-g_{2}}\right)$ vanish at the horizon, which in fact, corresponds to regular requirement of the physical quantities [11]. The electric current flux, required to cancel gauge anomaly and to demand gauge invariance at the quantum level to hold in the effective theory, then reads off

$$
c_{o}=-\frac{e^{2}}{2 \pi} A_{t}\left(r_{+}\right)
$$

In fact, the electric current flux is exactly equal to that of $(1+1)$-dimensional blackbody radiation with the Planck distribution, including a chemical potential for an electric charge $e$.

Next, we will study the flux of the energy momentum tensor. To demand general coordinate covariance at the quantum level, we expect that the flux is equal to that of Hawking radiation. When we exclude modes' propagation along the light-like direction at the horizon, in the region $r_{+}+\epsilon \leq r$ where the background contains a gauge potential but has no anomaly, the energy momentum tensor satisfies the modified conservation equation $\partial_{r}\left[\sqrt{-g_{2}} T_{t(o)}^{r}\right]=c_{o} \partial_{r} A_{t}$ deduced from the $\nu=t$ component of equation $\nabla_{\mu} T_{\nu}^{\mu}=0$, which is obeyed by the four-dimensional energy momentum tensor of the black hole. Near the horizon, the offending modes has been integrated out, the effective theory becomes chiral, and contains gauge and gravitational anomalies. Then the energy momentum tensor $T_{t}^{r}$ satisfies [11]

$$
\begin{aligned}
\partial_{r}\left[\sqrt{-g_{2}} T_{t(H)}^{r}\right] & =\sqrt{-g_{2}} J_{(H)}^{r} \partial_{r} A_{t} \\
& +A_{t} \partial_{r}\left[\sqrt{-g_{2}} J_{(H)}^{r}\right]+\partial_{r} N_{t}^{r}
\end{aligned}
$$

where, $N_{t}^{r}=\left(f_{, r} g_{, r}+g f_{, r r}\right) /(192 \pi)$. It is suffice for our discussions to only investigate the component $T_{t}^{r}$ since the anomaly is time-like. In the above equation, the second term comes from gauge anomaly while the third one is from gravitational anomaly. In the effective theory, since the energy momentum tensor combines contributions from both regions, that is, $T_{\nu}^{\mu}=$ $T_{\nu(H)}^{\mu} H(r)+T_{\nu(o)}^{\mu} \Theta_{+}(r)$, the effective action under general coordinate transformation changes as

$$
\begin{aligned}
-\delta W= & \int d t d r \lambda^{t}\left\{c_{o} \partial_{r} A_{t}+\partial_{r}\left[\left(\frac{e^{2}}{4 \pi} A_{t}^{2}+N_{t}^{r}\right) H(r)\right]\right. \\
+ & {\left[\sqrt{-g_{2}}\left(T_{t(o)}^{r}-T_{t(H)}^{r}\right)+\frac{e^{2}}{4 \pi} A_{t}^{2}+N_{t}^{r}\right] } \\
& \left.\times \delta\left(r-r_{+}-\epsilon\right)\right\} .
\end{aligned}
$$

where $\lambda^{t}$ is the general coordinate transformation parameter, and we have not incorporated the quantum effect of the ingoing modes. The first term is the classical effect of the background electric field for constant current flow, the second term is cancelled by the quantum effect of the ingoing modes whose contribution to the energy momentum tensor flux is $-\left[N_{t}^{r}+e^{2} A_{t}^{2} /(4 \pi)\right]$. To demand general coordinate symmetry of the effective action at the quantum level, the energy momentum tensor fluxes in both regions satisfy

$$
a_{o}=a_{H}+\frac{e^{2}}{4 \pi} A_{t}^{2}\left(r_{+}\right)-N_{t}^{r}\left(r_{+}\right),
$$

where

$$
\begin{aligned}
a_{o} & =\sqrt{-g_{2}} T_{(o) t}^{r}-c_{o} A_{t}(r), \\
a_{H} & =\sqrt{-g_{2}} T_{t(H)}^{r}-\int_{r_{+}}^{r} d r \partial_{r}\left(c_{o} A_{t}+\frac{e^{2} A_{t}^{2}}{4 \pi}+N_{t}^{r}\right),
\end{aligned}
$$

are the values of the energy flow at the infinity and the horizon, respectively.

Taking the form of the covariant energy momentum tensor as

$$
\sqrt{-g_{2}} \widetilde{T}_{t}^{r}=\sqrt{-g_{2}} T_{t}^{r}+\frac{g}{192 \pi f}\left(f f_{, r r}-2 f_{, r}^{2}\right)
$$

and further imposing the vanishing condition on it, which in fact corresponds to the regularity condition for the energy momentum tensor at the future horizon [11], one will find the flux of the energy momentum tensor, required to demand general coordinate covariance at the quantum level, is given by

$$
a_{o}=\frac{e^{2}}{4 \pi} A_{t}^{2}\left(r_{+}\right)+\frac{\pi}{12} T_{+}^{2},
$$

where

$$
\begin{aligned}
T_{+} & =\left.\frac{1}{4 \pi} \sqrt{f_{, r} g_{, r}}\right|_{r=r_{+}} \\
& =\frac{1}{4 \pi r_{+}}\left(1-\frac{r_{-}}{r_{+}}\right)^{\left(1-\alpha^{2}\right) /\left(1+\alpha^{2}\right)},
\end{aligned}
$$

is the Hawking temperature of the black hole. The energy momentum tensor flux exactly agrees with that of Hawking radiation from the black hole.

Finally let us study the electric current and energy momentum tensor fluxes of Hawking radiation in the case of fermions. For the charged black hole, the Hawking distribution for fermions is given by $N_{ \pm e}(\omega)=$ $1 /\left[\exp \left(\frac{\omega \mp e A_{t}\left(r_{+}\right)}{T_{+}}\right)+1\right]$, with which the electric current and energy momentum tensor fluxes have the completely equivalent forms to those obtained by the cancellation conditions of gauge and gravitational anomalies and the regularity requirement at the horizon [11, 12]. So, Hawking radiation can be derived from the anomalous point of view.

The spherically symmetric dilatonic black holes are derived from the low-energy string theory, and the background has a dilaton field coupled to a gauge field. The solutions have some interesting thermodynamical properties especially in the extreme case [16, 20], which are 
not found in the conventional black hole. The temperature of the spherically symmetric dilatonic black holes in the extreme limit depends drastically on the dilaton coupling constant $\alpha$. When the charge of the black hole approaches to the maximum value $Q_{\max }=\sqrt{1+\alpha^{2}} M$, the temperature of the black hole with $\alpha<1$ vanishes as it does in the Reissner-Nordström black hole case. If $\alpha>1$, it diverges in the extreme limit. When moving to the case $\alpha=1$, it has the nonzero finite value $1 /(8 \pi M)$. Besides, the energy momentum tensor flux for each value of the dilaton coupling constant $\alpha$ coincides at $Q=0$, since the black hole solution with any $\alpha$ is identically the Schwarzschild spacetime for $Q=0$ [19]; the difference becomes large as the charge increases. When the charge of the black hole is fixed, the charge current flux is eliminated from the anomalous flux. The energy momentum tensor flux in Eq. (14) is then nonzero but finite at $\alpha=1$ near the extreme limit. However, in the extreme case, it vanishes at $\alpha<1$, while the black hole with $\alpha>1$ radiates a large amount of energy. So, the static, spherically symmetric dilatonic black holes with the coupling constant $\alpha>1$ evolve rapidly into a naked singularity near the extreme case. In that case, we have to resort to the full quantum theory to properly study such problems.

\section{HAWKING RADIATION FROM THE ROTATING KALUZA-KLEIN DILATONIC BLACK HOLE}

In the rotating case, the Kaluza-Klein black hole is an exact solution to the action (1) with the coupling constant $\alpha=\sqrt{3}$. It is derived by a dimensional reduction of the boosted five-dimensional Kerr solution to four dimensions. The metric is given by [17, 19]

$$
\begin{aligned}
d s^{2}= & -\frac{\Delta-a^{2} \sin ^{2} \theta}{B \Sigma} d t^{2}-2 a \sin ^{2} \theta \frac{Z}{B \sqrt{1-\nu^{2}}} d t d \varphi \\
& +\left[B\left(r^{2}+a^{2}\right)+a^{2} \sin ^{2} \theta \frac{Z}{B}\right] \sin ^{2} \theta d \varphi^{2} \\
& +\frac{B \Sigma}{\Delta} d r^{2}+B \Sigma d \theta^{2},
\end{aligned}
$$

where

$$
\begin{aligned}
\Delta & =r^{2}-2 \mu r+a^{2}, & \Sigma & =r^{2}+a^{2} \cos ^{2} \theta, \\
Z & =\frac{2 \mu r}{\Sigma}, & B & =\sqrt{1+\frac{\nu^{2} Z}{1-\nu^{2}} .}
\end{aligned}
$$

The dilaton field is $\phi=-(\sqrt{3} / 2) \ln B$, and the potential corresponding to the gauge field is

$$
A_{t}=\frac{\nu Z}{2\left(1-\nu^{2}\right) B^{2}}, \quad A_{\varphi}=-\frac{a \nu Z \sin ^{2} \theta}{2 \sqrt{1-\nu^{2}} B^{2}} .
$$

The physical mass $M$, the charge $Q$ and the angular momentum $J$ are expressed, by the boost parameter $\nu$, mass parameter $\mu$ and specific angular momentum $a$, as

$$
\begin{aligned}
M & =\mu\left[1+\frac{\nu^{2}}{2\left(1-\nu^{2}\right)}\right], \\
Q & =\frac{\mu \nu}{1-\nu^{2}}, \quad J=\frac{\mu a}{\sqrt{1-\nu^{2}}},
\end{aligned}
$$

respectively. When $Q=0$, the black hole is reduced to Kerr black hole. The $J=0$ case corresponds to the non-rotating black hole with the same coupling constant $\alpha=\sqrt{3}$. The event horizon of the black hole is given by $r_{+}=\mu+\sqrt{\mu^{2}-a^{2}}$, and the nonsingular inner horizon is located at $r_{-}=\mu-\sqrt{\mu^{2}-a^{2}}$. The condition that the black hole has a regular horizon is $\mu^{2} \geq a^{2}$. It should be noticed that the solution, in the extreme limit $(|Q|=2 M$ and $J=0$ ), is no longer representing a black hole because a naked singularity appears [19]. This is quite different from the Kerr-Newman $(\alpha=0)$ black hole.

Now, we study Hawking radiation from the rotating Kaluza-Klein black hole via the anomalous point of view. Near the horizon, performing the partial wave decomposition of the scalar field in term of the spherical harmonics $\varphi=\sum_{l, m} \varphi_{l m}(t, r) Y_{l m}(\theta, \phi)$ [Strictly speaking, the angular part of the separated scalar field equation can be transformed into a form of confluent Heun equation [21], but near the horizon it approaches to the spherical harmonic since the near-horizon geometry has a topology of 2-sphere.] and transforming to the tortoise coordinate defined by $d r_{*} / d r=\left.B\right|_{\theta=0}\left(r^{2}+a^{2}\right) / \Delta \equiv 1 / f(r)$, one can easily observe that the effective two-dimensional theory, decided by the following metric and gauge field

$$
\begin{aligned}
d s^{2} & =-f(r) d t^{2}+f^{-1}(r) d r^{2}, \\
\mathcal{A}_{t} & =-\frac{Q r e\left(1-\nu^{2}\right)}{r^{2}+a^{2}}-\frac{a m \sqrt{1-\nu^{2}}}{r^{2}+a^{2}},
\end{aligned}
$$

is capable of describing the physics near the horizon of the higher-dimensional black hole. Here, we still omit the dilaton background due to the reduced static spacetime. In the gauge field background, $e$ and $m$ are the electric charge and azimuthal angular quantum number of the scalar field, respectively. The first term stems from the electric field of the charged black hole, and the second one is the induced gauge potential associated with the black hole's axisymmetry. So, the two-dimensional reduction includes general coordinate symmetry and two gauge symmetries. When omitting the classically irrelevant ingoing modes near the horizon, the effective action becomes anomalous with respect to these symmetries. In order to relieve the conflict between a symmetry of the classical action and the procedure of quantization, one must introduce the corresponding fluxes to cancel these anomalies.

As before, the first thing is to study gauge current flux and gauge anomalies. The effective field theory is defined outside the horizon. Near the horizon $r_{+} \leq r \leq r_{+}+\epsilon$, when excluding the horizon-skimming modes, the current $\widetilde{J}$ derived from the electric current in the original charged 
black hole, becomes anomalous and satisfies the anomalous equation [11] as $\partial_{r} \widetilde{J}_{(H)}^{r}=e \partial_{r} \mathcal{A}_{t} /(4 \pi)$. [In fact, the gauge potential corresponding to the electric charge $e$ in the two-dimensional reduction is given by $\widetilde{\mathcal{A}}_{t}=\mathcal{A}_{t} / e$, and the current should satisfy $\partial_{r} \widetilde{J}_{(H)}^{r}=e^{2} \partial_{r} \widetilde{\mathcal{A}}_{t} /(4 \pi)$.] In the other region, there is no anomalies and the gauge current satisfies the conservation equation $\partial_{r} \widetilde{J}_{(o)}^{r}=0$. Under gauge transformations, to demand the effective action gauge invariance at the quantum level, the compensating flux of the current, is then written as

$$
d_{o}=-\frac{e}{2 \pi} \mathcal{A}_{t}\left(r_{+}\right),
$$

where we have imposed the condition that the covariant gauge current, which is expressed by $\widehat{J}_{(H)}^{r}=\widetilde{J}_{(H)}^{r}+$ $e \mathcal{A}_{t}\left(r_{+}\right) /(4 \pi)$, vanishes at the future horizon [11, 12]. This result is in agreement with the electric current flux of Hawking radiation.

In addition to the energy and charge conservation, one should also take into account the conservation of angular momentum in the rotating Kaluza-Klein dilatonic black hole. In two-dimensional theory, the axisymmetry of the rotating black hole can now be interpreted as $U(1)$ gauge symmetry for the two-dimensional scalar field. There is an induced $U(1)$ gauge potential corresponding to this $U(1)$ gauge symmetry, whose gauge charge $m$ is an azimuthal angular momentum quantum number. When the effective field theory is defined outside the horizon, this $U(1)$ gauge current $\widehat{J}$, which is deduced from the $(r, \varphi)$-component of the four-dimensional energy momentum tensor, is composed of contributions from two regions. In the region $r_{+}+\epsilon \leq r$, there is no anomalies, and the gauge current satisfies the conservation equation $\partial_{r} \widehat{J}_{(o)}^{r}=0$. But near the horizon, it exhibits an anomaly with respect to $U(1)$ gauge symmetry since the offending modes are removed and the effective theory becomes chiral here, and satisfies the anomalous equation $\partial_{r} \widehat{J}_{(H)}^{r}=m \partial_{r} \mathcal{A}_{t} /(4 \pi)$, where the azimuthal quantum number $m$ is treated as the $U(1)$ gauge charge. Under gauge transformations, the $U(1)$ gauge current flux, required to cancel $U(1)$ gauge anomaly, reads off

$$
f_{o}=-\frac{m}{2 \pi} \mathcal{A}_{t}\left(r_{+}\right) \text {. }
$$

This factually corresponds to the angular momentum flux of Hawking radiation.

We now investigate the flux of the energy momentum tensor. In classical theory, the symmetry of the action under general coordinate transformations requires the covariant conservation of energy momentum tensor. But as mentioned before, there is a divergent energy momentum tensor near the horizon due to a pile up of the horizonskimming modes. Suppose that the effective field theory is formulated to exclude the offending modes, it no longer has a divergent energy momentum tensor near the horizon, but contains an anomaly with respect to general coordinate symmetry and takes the form of the nonconservation of energy momentum tensor. Adopting this picture, one can reformulate the effective field theory outside the horizon. In the region $r_{+}+\epsilon \leq r$, it contains all the same modes as the fundamental theory does, so there is no anomaly. The energy momentum tensor, under an effective background gauge field, satisfies the modified conservation equation $\partial_{r} T_{t(o)}^{r}=\mathcal{J}_{(o)}^{r} \partial_{r} \mathcal{A}_{t}$. However, it becomes anomalous near the horizon since the numbers of the outgoing modes and ingoing modes are no longer matched with each other when omitting the classically irrelevant ingoing modes. The energy momentum tensor near the horizon satisfies the anomalous equation

$$
\partial_{r} T_{t(H)}^{r}=\mathcal{J}_{(H)}^{r} \partial_{r} \mathcal{A}_{t}+\mathcal{A}_{t} \partial_{r} \mathcal{J}_{(H)}^{r}+\partial_{r} N_{t}^{r},
$$

where the current is defined by $\mathcal{J}^{r} \equiv \widetilde{J}^{r} / e=\widehat{J}^{r} / m$, and $N_{t}^{r}=\left(f_{, r}^{2}+f f_{, r r}\right) /(192 \pi)$. Imposing the boundary conditions that the covariant energy momentum tensor, defined by $\widehat{T}_{t}^{r}=T_{t}^{r}+\left(f f_{, r r}-2 f_{, r}^{2}\right) /(192 \pi)$, vanishes at the horizon and there is no ingoing modes at radial infinity, the covariance of the effective theory at the quantum level, under general coordinate transformations, requires a compensating flux of the energy momentum tensor

$$
g_{o}=\frac{1}{4 \pi} \mathcal{A}_{t}^{2}\left(r_{+}\right)+\frac{\pi}{12} T_{+}^{2},
$$

where

$$
T_{+}=\left.\frac{1}{4 \pi} \partial_{r} f\right|_{r=r_{+}}=\frac{\sqrt{1-\nu^{2}} \sqrt{\mu^{2}-a^{2}}}{2 \pi\left(r_{+}^{2}+a^{2}\right)},
$$

is the Hawking temperature of the black hole. In fact, the energy momentum tensor flux is exactly equal to that of Hawking radiation.

As for the fermionic case, the Planck distribution (including the chemical potentials for an azimuthal angular quantum number $m$ and an electric charge $e$ ), the electric potential and the angular velocity at the black hole horizon are, respectively, given by

$$
\begin{gathered}
N_{ \pm e, \pm m}(\omega)=1 /\left[\exp \left(\omega \mp e \Phi_{+} \mp m \Omega_{+}\right)+1\right], \\
\Phi_{+}=\frac{Q r_{+}\left(1-\nu^{2}\right)}{r_{+}^{2}+a^{2}}=\frac{\nu}{2}, \quad \Omega_{+}=\frac{a \sqrt{1-\nu^{2}}}{r_{+}^{2}+a^{2}},
\end{gathered}
$$

the electric current, angular momentum, and energy momentum tensor fluxes of Hawking radiation have the forms equivalent to those required to cancel gauge and gravitational anomalies and to demand gauge invariance and general coordinate covariance at the quantum level to hold in the effective theory [11]. Thus, Hawking radiation can be effectively described from the anomalous point of view.

Now, we study the properties of the derived physical quantities in the extreme case. Obviously, the temperature of the rotating dilatonic black hole vanishes in the extreme limit $\mu=a$. In addition, when taken the limit $Q \rightarrow Q_{\max }=2 M$, keeping the black hole extreme with 
$J \neq 0$ (whereas $J \rightarrow 0$ in the extreme), the value of the temperature is still zero, which is different from the nonrotating dilatonic black holes, where the temperature diverges in the limit $Q \rightarrow Q_{\max }=2 M$. Thus the temperature is discontinuous at $Q=Q_{\max }$; this is due to the fact that there is a naked singularity for the Klauza-Klein black hole when reaching to the extreme case. Moreover, the angular velocity $\Omega$, under the limit $Q \rightarrow Q_{\max }$, diverges at the horizon, but the angular momentum $J$ vanishes. This is because the horizon shrinks to zero size as the angular momentum decreases. The solution with $Q \rightarrow Q_{\max }$ then represents a rapidly spinning black hole [19].

Subsequently, we will vary the charge to see how the angular momentum and energy momentum tensor fluxes change in the maximally charged limit. As for the rotating Kaluza-Klein dilatonic black hole, the temperature is zero in the extreme case, and the thermal emission vanishes. However, in the maximally charged limit $Q \rightarrow Q_{\max }$, while the angular momentum itself is small, the angular velocity of the black hole is very large and its superradiance effect becomes important. The angular momentum and energy momentum tensor fluxes increase rapidly till the black hole approaches to the maximally charge state, where the horizon radius is smaller than the Planck scale. However a better understanding of physics at the Planck scale is a prerequisite; this is especially necessary for studying black holes at the maximally charge state, which have to rely on the full quantum theory.

\section{HAWKING RADIATION FROM THE ROTATING KERR-SEN DILATONIC BLACK HOLE}

The Kerr-Sen black hole [18] is a solution to the lowenergy effective action in heterotic string theory. The action containing a three-form (axion) field $H$ and a dilaton field $\phi$ coupled to a $U(1)$ gauge field $F$ reads off $[18,19]$

$S=\frac{1}{16 \pi} \int d^{4} x \sqrt{-g}\left[R-2(\nabla \phi)^{2}-e^{-2 \phi} F^{2}-\frac{1}{12} e^{-4 \phi} H^{2}\right]$.

From the uncharged Kerr solution, Sen [18] adopted the solution generating technique to obtain a new rotating one, which is given by

$$
\begin{aligned}
d s^{2}= & -\frac{\Delta-a^{2} \sin ^{2} \theta}{\Sigma} d t^{2}-\frac{4 \mu r a \cosh ^{2} \beta \sin ^{2} \theta}{\Sigma} d t d \varphi \\
& +\frac{\Lambda \sin ^{2} \theta}{\Sigma} d \varphi^{2}+\frac{\Sigma}{\Delta} d r^{2}+\Sigma d \theta^{2}
\end{aligned}
$$

where

$$
\begin{aligned}
\Sigma \equiv & r^{2}+a^{2} \cos ^{2} \theta+2 \mu r \sinh ^{2} \beta, \\
\Delta \equiv & r^{2}-2 \mu r+a^{2}, \\
\Lambda \equiv & \left(r^{2}+a^{2}\right)\left(r^{2}+a^{2} \cos ^{2} \theta\right)+2 \mu r a^{2} \sin ^{2} \theta \\
& +4 \mu r\left(r^{2}+a^{2}\right) \sinh ^{2} \beta+4 \mu^{2} r^{2} \sinh ^{4} \beta .
\end{aligned}
$$

The dilaton field, axion field and gauge potential corresponding to the gauge field are, respectively, given by

$$
\begin{aligned}
& \phi=\frac{-1}{2} \ln \frac{\Sigma}{r^{2}+a^{2} \cos ^{2} \theta}, B_{t \varphi}=2 a \sin ^{2} \theta \frac{\mu r \sinh ^{2} \beta}{\Sigma}, \\
& A_{t}=\frac{\mu r \sinh 2 \beta}{\sqrt{2} \Sigma}, \quad A_{\varphi}=-a \sin ^{2} \theta \frac{\mu r \sinh 2 \beta}{\sqrt{2} \Sigma} .
\end{aligned}
$$

The mass $M$, the charge $Q$, and the angular momentum $J$ are given by the parameters $\mu, \beta$ and $a$ as

$$
\begin{aligned}
M & =\frac{\mu}{2}(1+\cosh 2 \beta), \\
Q & =\frac{\mu}{\sqrt{2}} \sinh 2 \beta, \quad J=M a .
\end{aligned}
$$

The horizon radius is determined as $r_{+}=\mu+\sqrt{\mu^{2}-a^{2}}$. The condition for the solution to be a black hole is $\mu \geq$ $|a|$, which can be rewritten as

$$
|J| \leq M^{2}-Q^{2} / 2
$$

In the extreme limit $|Q|=\sqrt{2} M$ and $J=0$, a naked singularity appears, so the solution is no longer a black hole [19].

Following the reduction procedure adopted above, the physics near the horizon in higher dimensional black hole can be effectively described by a two-dimensional theory. In the present case, each partial wave in the near-horizon limit behaves as an independent two dimensional scalar field in the background of the dilaton field whose contributions are omitted due to the static background. The two-dimensional metric and the gauge field are

$$
\begin{aligned}
d s^{2} & =-f(r) d t^{2}+f^{-1}(r) d r^{2} \\
\widetilde{\mathcal{A}}_{t} & =-\frac{Q r e}{\left(r^{2}+a^{2}\right) \cosh ^{2} \beta}-\frac{2 \mu a r}{\left(r^{2}+a^{2}\right)^{2} \cosh ^{2} \beta} .
\end{aligned}
$$

where, $f(r)=\Delta /\left(r^{2}+a^{2}+2 \mu r \sinh ^{2} \beta\right)$. Apparently, the two-dimensional theory for each partial wave has two gauge symmetries. If an effective field theory is formulated outside the horizon to integrate out the horizonskimming modes, the electric current, angular momentum, and energy momentum tensor become anomalous with respect to gauge and general coordinate symmetries near the horizon. To demand gauge invariance and general coordinate covariance at the quantum level to hold in the effective theory, one must introduce the compensating electric current, angular momentum, and energy momentum tensor fluxes to cancel these anomalies at the horizon, and they are precisely equal to those of Hawking radiation.

As usual, we will first study the electric current flux and its gauge anomaly. The effective theory is still formulated outside the horizon to integrate out the horizonskimming modes. Near the horizon, it becomes chiral and contains gauge anomaly. Under gauge transformations, the electric current exhibits an anomaly with respect 
to gauge symmetry, whose consistent form satisfies the anomalous equation $\partial_{r} j_{(H)}^{r}=e \partial_{r} \widetilde{\mathcal{A}}_{t} /(4 \pi)$. In the other region where the effective theory contains all modes, the current satisfies the conservation equation $\partial_{r} j_{(o)}^{r}=0$, in which $j$ is derived from the electric current in the fourdimensional spacetime. Under gauge transformations, to demand the effective action gauge invariance at the quantum level, the electric current describes a flux given by

$$
h_{o}=-\frac{e}{2 \pi} \widetilde{\mathcal{A}}_{t}\left(r_{+}\right),
$$

where we have already imposed the condition that the covariant form of the current vanishes at the horizon [11, 12]. This electric current flux, derived from gauge anomaly cancellation and the regularity requirement at the horizon, is exactly equal to that of Hawking radiation.

Similarly, we can derive the flux of the $U(1)$ gauge current. The gauge current $\widetilde{j}$, deduced from the component of the four-dimensional energy momentum tensor $T_{\varphi}^{r}$, becomes anomalous with respect to $U(1)$ gauge symmetry when excluding the offending modes near the horizon. In the two-dimensional theory, the $U(1)$ gauge current near the horizon satisfies the anomalous equation $\partial_{r} \widetilde{j}_{(H)}^{r}=m \partial_{r} \widetilde{\mathcal{A}}_{t} /(4 \pi)$. However, it is conserved $\partial_{r} \widetilde{j}_{(o)}^{r}=0$ in the region $r_{+}+\epsilon \leq r$. The $U(1)$ gauge current flux, required to cancel $U(1)$ gauge anomaly, is then given by

$$
i_{o}=-\frac{m}{2 \pi} \widetilde{\mathcal{A}}_{t}\left(r_{+}\right),
$$

where we have assumed the vanishing condition of the covariant $U(1)$ gauge current at the horizon. This angular momentum flux exactly corresponds to that of Hawking radiation.

The above two gauge anomalies stem from the destruction of gauge symmetries associated with the electric field and the axisymmetry of the rotating charged black hole. Besides these, gravitational anomaly in general coordinate symmetry appears in the two-dimensional background when we omit the quantum effect of the ingoing modes. This is formally reflected by the nonconservation of the energy momentum tensor. In the following, the energy momentum tensor flux, required to restore general coordinate covariance at the quantum level, is derived from the anomalous point of view. We expect that the value of the energy momentum tensor flux is equal to that of Hawking radiation similar to the cases of the two gauge current fluxes. Near the horizon, the energy momentum tensor satisfies the anomalous equation (10) with the replacements $N_{t}^{r}=\left(f_{, r}^{2}+f f_{, r r}\right) /(192 \pi)$ and $A_{t}=\widetilde{\mathcal{A}}_{t}$. In the other region, there is no anomaly, but it contains a gauge field, the energy momentum tensor satisfies the modified conservation equation $\partial_{r} T_{t(o)}^{r}=\mathcal{J}_{(o)}^{r} \partial_{r} \widetilde{\mathcal{A}}_{t}$. In both regions, the current is defined by $\mathcal{J}^{r} \equiv j^{r} / e=$ $\widetilde{j}^{r} / m$. Imposing the condition that the covariant energy momentum tensor vanishes at the horizon, the energy momentum tensor flux, required to demand the effective action general coordinate covariance at the quantum level, is expressed by

$$
k_{o}=\frac{1}{4 \pi} \widetilde{\mathcal{A}}_{t}^{2}\left(r_{+}\right)+\frac{\pi}{12} T_{+}^{2},
$$

where $T_{+}$is the Hawking temperature of the black hole, whose obvious expression is given by

$$
T_{+}=\left.\frac{1}{4 \pi} \partial_{r} f(r)\right|_{r=r_{+}}=\frac{\sqrt{\mu^{2}-a^{2}}}{2 \pi\left(r_{+}^{2}+a^{2}\right) \cosh ^{2} \beta} .
$$

This fits in with the energy momentum tensor flux of blackbody radiation at the Hawking temperature with Planck distribution, including chemical potentials for an electric charge $e$ and an azimuthal quantum number $m$.

If we introduce the Planck distribution for fermions in the Kerr-Sen black hole as that in previous section, and replace the electric potential by $\Phi_{+}=Q /(2 M)=$ $Q r_{+} /\left[\left(r_{+}^{2}+a^{2}\right) \cosh ^{2} \beta\right]$, and the angular velocity by $\Omega_{+}=a /\left[\left(r_{+}^{2}+a^{2}\right) \cosh ^{2} \beta\right]$, the electric current, angular momentum, and energy momentum tensor fluxes of Hawking radiation still have the equivalent forms to those derived from anomaly cancellation condition and the regularity requirement at the horizon.

In the extreme limit $\mu=a$, the temperature and the angular velocity are no longer zero or divergent but approach to finite values, however they still discontinue at the maximally charged limit $Q=Q_{\max }=\sqrt{2} M$, since there exists a naked singularity for the extreme black hole. The angular momentum and energy momentum tensor fluxes of the Kerr-Sen black hole increase rapidly, but slower than those of the Kaluza-Klein black hole, towards the maximally charged limit. The final state is the presence of a naked singularity and the surface of the black hole vanishes, which means that we have to deal with a horizon radius smaller that the Planck scale. That needs to be investigated in the full quantum theory.

\section{DISCUSSIONS AND CONCLUSIONS}

In summary, we have applied the method of cancellation of anomaly to derive Hawking radiation of various dilatonic black holes. After reductions from the fourdimension theory, the effective $(1+1)$-dimensional field theory is formulated outside the horizon, which is largely based upon the choice of the vacuum state, namely the Unruh vacuum rather than the Boulware state. Excluding the offending modes at the horizon results in a breakdown at the quantum level of gauge invariance and general coordinate covariance. To demand these symmetries to hold in the effective theory, the compensating fluxes can be obtained by the anomaly cancellation and the regularity requirement at the horizon. In all cases, we find that the charge and energy momentum tensor fluxes, required to cancel the gauge and gravitational anomalies, 
are exactly in agreement with those of $(1+1)$-dimensional blackbody radiation at the Hawking temperature with Planck distribution including appropriate chemical potentials.

It should be emphasized that in the cases of rotating and charged dilatonic black holes, the RobinsonWilczek's proposal to derive Hawking radiation can be also verified in the dragging coordinate system. In a rotating spacetime, the matter field in the ergosphere near the horizon must be dragged by the gravitational field with an azimuthal angular momentum because there exists a frame dragging effect of the coordinate. In the dragging coordinate system, the matter field is co-rotating with the black hole, so the $U(1)$ gauge symmetry induced from the azimuthal symmetry no longer needs to be incorporated in the two-dimensional theory for each partial wave, and the corresponding gauge anomaly is excluded from this effective theory. To demand gauge invariance at the quantum level to hold in the original underlying theory, the compensating flux of the gauge current is only attributed to the contributions of gauge anomaly originated from the electric field of the original charged black hole. The fluxes determined by the anomaly cancellations and the regularity requirements at the horizon still have the completely equivalent forms to those of black- body radiation with Planck distribution function in the dragging coordinate.

In addition, the derivation of Hawking radiation via anomalies is based upon quantum field theory on the fixed background spacetime without considering the fluctuation of spacetime geometry. With the evaporation, the mass, the charge and the angular momentum of the realistic black hole must diminish and the background geometry must vary accordingly. Thus a bigger challenge is to incorporate the self-gravitation correction into the framework. Finally, in the maximally charged limit, the area of the charged dilatonic black hole vanishes, but the fluxes of the angular momentum and energy momentum tensor are still very large. To properly study Hawking radiation in these cases, the back reaction of the quantum effects must be taken into account and one has to resort to the full quantum theory.

Acknowledgments: S.-Q.Wu was partially supported by the Natural Science Foundation of China under Grant No. 10675051 and by a starting fund from Central China Normal University. X.Cai was supported in part by the NSFC of China (No. 70571027, No. 10635020) and by the Ministry of Education of China under Grant No. 306022 .
[1] S. Hawking, Nature (London) 248, 30 (1974); Commun. Math. Phys. 43, 199 (1975).

[2] M.K. Parikh and F. Wilczek, Phys. Rev. Lett. 85, 5042 (2000); Q.Q. Jiang, S.Q. Wu, and X. Cai, Phys. Rev. D 73, 064003 (2006); Erratum-ibid. 73, 069902 (2006).

[3] S. Hemming and E. Keski-Vakkuri, Phys. Rev. D 64, 044006 (2001); A.J.M. Medved, Phys. Rev. D 66, 124009 (2002); E.C. Vagenas, Phys. Lett. B 559, 65 (2003); Q.Q. Jiang and S.Q. Wu, Phys. Lett. B 635, 151 (2006); Erratum-ibid. 639, 684 (2006); hep-th/0603082

[4] K. Srinivasan and T. Padmanabhan, Phys. Rev. D 60, 024007 (1999); S. Shankaranarayanan, K. Srinivasan, and T. Padmanabhan, Mod. Phys. Lett. A 16, 571 (2001); S. Shankaranarayanan, T. Padmanabhan, and K. Srinivasan, Class. Quantum Grav. 19, 2671 (2002); T. Padmanabhan, Mod. Phys. Lett. A 19, 2637 (2004); M. Angheben, M. Nadalini, L. Vanzo, and S. Zerbini, J. High Energy Phys. 05, 014 (2005).

[5] S. Christensen and S.A. Fulling, Phys. Rev. D 15, 2088 (1977).

[6] R. Balbinot, A. Fabbri, and I. Shapiro, Phys. Rev. Lett. 83, 1494 (1999).

[7] S.P. Robinson and F. Wilczek, Phys. Rev. Lett. 95, 011303 (2005).

[8] W.G. Unruh, Phys. Rev. D 10, 3194 (1974).

[9] D.G. Boulware, Phys. Rev. D 11, 1404 (1975).

[10] S. Iso, H. Umetsu, and F. Wilczek, Phys. Rev. Lett. 96, 151302 (2006).
[11] S. Iso, H. Umetsu, and F. Wilczek, Phys. Rev. D 74, 044017 (2006).

[12] K. Murata and J. Soda, Phys. Rev. D 74, 044018 (2006).

[13] E.C. Vagenas and S. Das, J. High Energy Phys. 10, 025 (2006); M.R. Setare, The European Physical Journal C 49, 865 (2007); Z. Xu and B. Chen, Phys. Rev. D 75 024041 (2007); S. Iso, T. Morita, and H. Umetsu, JHEP 04 (2007) 068; Q.Q. Jiang and S.Q. Wu, Phys. Lett. B 647, 200 (2007); Q.Q. Jiang, S.Q. Wu, and X. Cai, hep-th/0701048

[14] G.W. Gibbons and K.I. Maeda, Nucl. Phys. B 298, 741 (1988).

[15] D. Garfinkle, G.T. Horowitz, and A. Strominger, Phys. Rev. D 43, 3140 (1991); Erratum-ibid. 45, 3888 (1992);

[16] J.H. Horne and G.T. Horowitz, Phys. Rev. D 46, 1340 (1992).

[17] V.P. Frolov, A.I. Zelnikov, and U. Bleyer, Ann. Physik (Leipzig) 44, 371 (1987); G.W. Gibbons and D.L. Wiltshire, Ann. Phys. (N.Y.) 167, 201 (1986).

[18] A. Sen, Phys. Rev. Lett. 69, 1006 (1992).

[19] J.I. Koga and K.I. Maeda, Phys. Rev. D 52, 7066 (1995).

[20] A. Ghosh and P. Mitra, Phys. Rev. Lett. 73, 2521 (1994); D.J. Loranz and W.A. Hiscock, Phys. Rev. D 55, 3893 (1997).

[21] S.Q. Wu and X. Cai, J. Math. Phys. 40, 4538 (1999); 44, 1084 (2003). 